\title{
Correspondence
}

Korean J Ophthalmol 2020;34(4):334-335 https://doi.org/10.3341/kjo.2020.0016

\section{A Rare Complication of a Large Em- physema after Orbital Wall Fracture Surgery with Silastic Sheet Implant}

\section{Dear Editor,}

Implants, the mainstay of treatment for repair of orbital wall fractures, restore the structure and function of the internal orbit. Inflammation and infections related to implants are common complications of orbital wall fracture repair. Most complications occur acutely, with immediate rejection occurring within the first few months after surgery. However, there have been a few reported cases of delayed infections that were cystic in form. Here, we present a case of delayed emphysema in the medial orbital wall of a patient who underwent repair 15 years previously. To our knowledge, this is the first case report of an encapsulated emphysema resulting from a delayed inflammatory reaction to a silastic sheet implant.

A 43-year-old female presented to our clinic complaining of recurrent swelling of the left medial canthal area for 3 years. Her past ocular history was remarkable for a traumatic left orbital wall fracture repair with a silastic sheet 15 years previously. Upon presentation, her best-corrected visual acuity was $20 / 20$ in both eyes, and intraocular pressure was $14 \mathrm{mmHg}$ in the right and $12 \mathrm{mmHg}$ in the left. Hertel exophthalmometry was $14 \mathrm{~mm}$ on the right and $16 \mathrm{~mm}$ on the left with a base of $113 \mathrm{~mm}$. Hess and the binocular single vision test showed no specific findings. No definite limitation of ocular motility or strabismus was noted in primary position. Furthermore, no signs of inflammation (heat sensation, redness, and tenderness) were

Received: February 12, 2020 Final revision: May 5, 2020 Accepted: May 6, 2020 noted. Computed tomography (CT) revealed an encapsulated emphysema surrounding a silastic sheet displaced into the ethmoid sinus, shifting the left eyeball temporally (Fig. 1A, 1B). The fovea ethmoidalis was severely eroded with suspicious extrusion of the silastic sheet. The patient underwent surgical removal of the implant with partial removal of the fibrous capsule through a transcaruncle and fornix approach. Intraoperative findings revealed an intact lacrimal sac and absence of cerebrospinal fluid leakage. Pathology of the capsule revealed granulation and fibrosis, and culture results were negative. We believe that a capsule formed around the silastic sheet, resulting in an airway connection to the sinus and allowed entrapment of air. A part of the fibrous capsule was intentionally left in place to prevent enophthalmos (Fig. 1C, 1D). Follow-up evaluation showed no complications, and a one-year postoperative CT
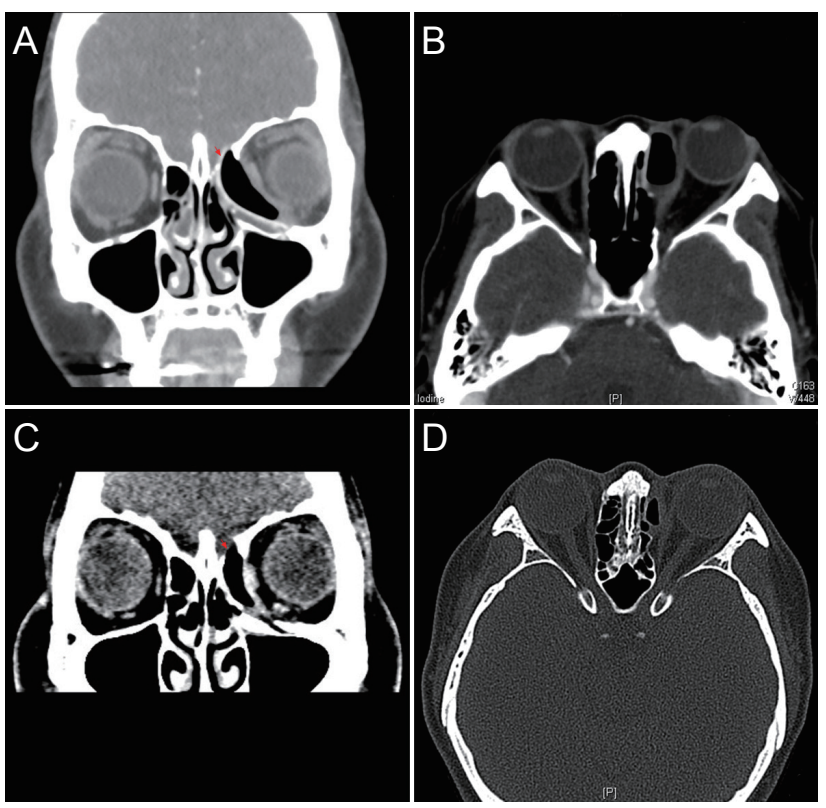

Fig. 1. Preoperative and postoperative computed tomography scan of the patient. (A,B) An encapsulated emphysema associated with a slipped silastic sheet implant is noted. $(\mathrm{C}, \mathrm{D})$ A computed tomography scan after the removal of the implant shows good postoperative results. The patient provided written informed consent for the use of these images. 
scan revealed no significant findings. With good postoperative results, she was lost to follow-up.

To date, several alloplastic materials, such as silastic sheeting, titanium, and porous polyethylene, have been introduced. These materials eliminate donor site morbidity, reduce operative time, and are readily available. Silastic sheets have been favored in the past for their low cost, flexibility, and durability in large orbital fractures. However, post-operative complications, such as fibrous capsule formation, infection, and extrusion, have been reported. In such cases, silastic sheets can easily be removed due to absence of adhesion and vascularization. Nevertheless, surgical results using silastic sheets are controversial. Morrison et al. [1] reported that $13.2 \%$ of patients underwent implant removal due to infection, pain, extrusion, or diplopia. Yun et al. [2] reported that $4.3 \%$ of patients required implant removal within a mean of 23.3 months. Moon et al. [3] reported that the revision rate in groups using silastic plates was $5.4 \%$, which was significantly higher than the rate for other materials. However, Prowse et al. [4] reported that the revision rate following use of silastic sheets was lower than that rate for non-silastic materials. Currently, our institute no longer uses silastic sheets for fracture reconstruction. Instead, titanium and porous polyethylene are used.

In the present case, swelling of the medial canthal area was the patient's only clinical symptom. The encapsulated emphysema caused displacement of the eyeball and thinning of the ethmoid roof, threatening extrusion into the cranial space. Capsule formation near the medial orbital wall is extremely dangerous and significantly increases the risk of infection due to anatomical proximity to the ethmoid sinus. We recommend a CT scan for all patients with history of wall fracture repair who present with symptoms of swelling despite absence of signs of inflammation. In our case, timely removal of the implant with partial removal of the fibrous capsule prevented severe complications. As new alloplastic materials emerge in clinical practice, the role of the ophthalmologist will become increasingly important for early recognition and timely management of unprecedented adverse events.

Ella SeoYeon Park, Bo Ram Kim, Jae Sang Ko, Jin Sook Yoon

Department of Ophthalmology, Institute of Vision Research, Severance Hospital, Yonsei University College of Medicine, Seoul, Korea

E-mail (Jin Sook Yoon): yoonjs@yuhs.ac

\section{Conflict of Interest}

No potential conflict of interest relevant to this article was reported.

\section{References}

1. Morrison AD, Sanderson RC, Moos KF. The use of silastic as an orbital implant for reconstruction of orbital wall defects: review of 311 cases treated over 20 years. J Oral Maxillofac Surg 1995;53:412-7.

2. Yun HK, Yi S, Joh SP, et al. Complications after open reduction of blow-out fracture using silicone sheet. $J$ Korean Cleft Palate-Craniofac Assoc 2001;2:72-6.

3. Moon SJ, Suh HS, Park BY, Kang SR. Safety of silastic sheet for orbital wall reconstruction. Arch Plast Surg 2014;41:362-5.

4. Prowse SJ, Hold PM, Gilmour RF, et al. Orbital floor reconstruction: a case for silicone: a 12 year experience. $J$ Plast Reconstr Aesthet Surg 2010;63:1105-9. 